\title{
A Supersymmetric Transfer Matrix and Differentiability of the Density of States in the One-Dimensional Anderson Model
}

\author{
Massimo Campanino ${ }^{\star}$ and Abel Klein $\star \star$ \\ Department of Mathematics, University of California, Irvine, Ca 92717, USA
}

\begin{abstract}
Let $H=-\Delta+V$ on $l^{2}(\mathbb{Z})$, where $V(x), x \in \mathbb{Z}$, are i.i.d.r.v.'s with common probability distribution $v$. Let $h(t)=\int e^{-i t v} d v(v)$ and let $k(E)$ be the integrated density of states. It is proven: (i) If $h$ is $n$-times differentiable with $h^{(j)}(t)=O\left((1+|t|)^{-\alpha}\right)$ for some $\alpha>0, j=0,1, \ldots, n$, then $k(E)$ is a $C^{n}$ function. In particular, if $v$ has compact support and $h(t)=O\left((1+|t|)^{-\alpha}\right)$ with $\alpha>0$, then $k(E)$ is $C^{\infty}$. This allows $v$ to be singular continuous. (ii) If $h(t)=O\left(e^{-\alpha|t|}\right)$ for some $\alpha>0$ then $k(E)$ is analytic in a strip about the real axis.

The proof uses the supersymmetric replica trick to rewrite the averaged Green's function as a two-point function of a one-dimensional supersymmetric field theory which is studied by the transfer matrix method.
\end{abstract}

\section{Introduction}

The one-dimensional Anderson model is given by the random Hamiltonian $H=$ $H_{0}+V$ on $l^{2}(\mathbb{Z})$, where

$$
\left(H_{0} u\right)(x)=\frac{1}{2}(u(x+1)+u(x-1))
$$

and $V(x), x \in \mathbb{Z}$, are independent identically distributed random variables with common probability distribution $v$. We will denote by $h$ its characteristic function, i.e., $h(t)=\int e^{-i t v} d v(v)$.

Let $\Lambda$ be an interval in $\mathbb{Z}$, we will denote by $H_{\Lambda}$ the operator $H$ restricted to $l^{2}(\Lambda)$ with boundary condition $u(x)=0$ for $x$ not in $\Lambda$.

The integrated density of states, $k(E)$, is defined by

$$
k(E)=\lim _{|\Lambda| \rightarrow \infty} \#\left\{\text { eigenvalues of } H_{\Lambda} \leqq E\right\} .
$$

* Permanent address: Dipartimento di Matematica, II Universita di Roma, Via Orazio Raimondo, I-00173 (La Romanina) Roma, Italy

$\star \star$ Research partially supported by the NSF under grant MC-8301889 
It is a consequence of the ergodic theorem that for almost every potential the limit exists for all $E$ and is independent of the potential [14]; $k(E)$ is always a continuous function [5]. Under some mild condition on $v k(E)$ was shown to be log-Holder continuous [6] and Holder continuous on compact intervals [7].

Without restrictions on $v$ we cannot expect too much more regularity. There is an argument of Halperin (see [8]) that shows that when $v=\frac{1}{2} \delta(v)+\frac{1}{2} \delta(v-a)$, given any $\alpha>0$ one can choose a so that $k(E)$ is not Holder continuous of order $\alpha$; in particular it gives examples where $k(E)$ is not $C^{1}$.

Further results have required $v$ to be absolutely continuous with respect to Lebesgue measure, say $d v(v)=F(v) d v$. If $F$ is bounded, Wegner [9] proved that $k(E)$ is absolutely continuous with a bounded derivative. This has been extended by Maier [10] to $F \in L^{p}, p>1$. If $\int v^{2} F(v) d v<\infty$, Lacroix [11] has shown $k(E)$ is $C^{1}$.

Constantinescu, Fröhlich and Spencer [12] proved that if $F$ is analytic in a strip of certain width, then $k(E)$ is real analytic for $|E|$ large enough; If $v$ is Gaussian they proved that for large disorder $k(E)$ is a real analytic function of $E$. Carmona [4], using an idea of Molcanov, gives a simple proof that if $|h(t)| \leqq C^{\prime} e^{-C|t|}$, where $C^{\prime}<C$, then $k(E)$ is analytic in a strip; this holds for $v$ Gaussian for large disorder. Another argument for the same result due to Simon can be found in [12].

Using the supersymmetric replica trick and a cluster expansion Klein and Perez [13] showed how to use decay properties of $h(t)$ and its derivatives to derive differentiability for $k(E)$ for either large disorder or large $|E|$; they also obtained analyticity results. Their methods have strongly influenced this article.

Recently, Simon and Taylor [8] proved the surprising (at least at first sight) result that if $d v(v)=F(v) d v$, where $F$ has compact support and $F \in L_{\alpha}^{1}(\mathbb{R})=\left\{f \in L^{1}(\mathbb{R}) \mid\right.$ there exists $g \in L^{1}(\mathbb{R})$ such that $\left.\hat{g}(t)=\left(1+t^{2}\right)^{\alpha / 2} \hat{f}(t)\right\}$, with $\alpha>0$, then $k(E)$ is $C^{\infty}$. They also conjectured that it should be enough to require that $\left(1+t^{2}\right)^{\alpha / 2} h(t)$ be bounded for some $\alpha>0$, and that the hypothesis of compact support should not be essential. As they remarked, there are singular continuous $v$ satisfying this condition (see [27, Theorem XII.10.12] and [28]).

In this article we prove Simon and Taylor's conjecture. We also prove analyticity results for the density of states.

Our condition will be stated in terms of $h$, the characteristic function of $v$. We will only be interested in $h(t)$ for $t \geqq 0$ (of course, $h(-t)=\overline{h(t)}$ ) and we will only consider the right-hand side derivatives at $t=0$.

We will now state our results.

Theorem 1.1. Let $n \geqq 1$. If $h$ is $(n-1)$-times differentiable for $t \geqq 0$ with $h^{(n-1)}$ absolutely continuous, and $(1+|t|)^{\alpha} h^{(j)}(t)$ is bounded for some $\alpha>0$ and $j=0,1$, $2, \ldots, n$, then $k(E)$ is a $C^{n}$ function of $E$.

Corollary 1.2. Let $(1+|t|)^{\alpha} h(t)$ be bounded for some $\alpha>0$. If $\int|v|^{n+\varepsilon} d v(v)<\infty$ for some $\varepsilon>0 k(E)$ is $C^{n}$. In particular, if $v$ has finite moments of all orders $k(E)$ is $C^{\infty}$.

Our result on analyticity is

Theorem 1.3. If $e^{\alpha \mid t} h(t)$ is bounded for some $\alpha>0$ then $k(E)$ is analytic in a strip $|\operatorname{Im} E|<\alpha_{1}$ for some $\alpha_{1}>0$.

We approach the density of states thru the Green's function of $H$. Let $G(x, y ; z)=$ 
$\left\langle x\left|(H-z)^{-1}\right| y\right\rangle$ where $x, y \in \mathbb{Z}, \operatorname{Im} z>0$. Then (e.g., $\left.[4,14]\right) G(z)=\mathbb{E}(G(0,0 ; z))$ is the Borel transform of $d k(E)$, i.e.,

$$
G(z)=\int \frac{d k(E)}{E-z}
$$

and we have:

i) $G(E+i 0)=\lim _{\eta \downarrow 0} G(E+i \eta)$ exists for a.e. $E \in R$,

ii) if $d k_{\text {a.c. }}$ denotes the absolutely continuous part of the measure $d k$,

$$
\frac{d k_{\text {a.c. }}}{d E}=\frac{1}{\pi} \operatorname{Im} G(E+i 0)
$$

iii) $d k_{\text {sing }} \equiv d k-d k_{\text {a.c. }}$ is supported by

$$
\{E \in R \mid \lim \operatorname{Im} G(E+i \eta)=\infty\} \text {. }
$$

Thus Theorem 1.1 and 1.3 follow from

Theorem 1.4. Let $n \geqq 1$. If $h$ is $(n-1)$-times differentiable for $t \geqq 0$ with $h^{(n-1)}$ absolutely continuous and $(1+|t|)^{\alpha} h^{(j)}(t)$ is bounded for some $\alpha>0$ and all $j=0$, $1, \ldots, n$, then $G(E+i 0)=\lim _{\eta \downarrow 0} G(E+i \eta)$ exists for all $E \in R$ and is a $C^{n-1}$ function of $E$.

Theorem 1.5. If $e^{\alpha|t|} h(t)$ is bounded for some $\alpha>0$ then $G(z)$ has an analytic continuation to $\operatorname{Im} z+\alpha_{1}>0$ for some $\alpha_{1}>0$.

We will now describe the strategy of our proof. Let $\Lambda_{l}=\{-l,-l+1, \ldots, 0, \ldots, l\}$, $H_{l}=H_{\Lambda_{l}}$, and

$$
G_{l}(z)=\mathbb{E}\left(\left\langle 0\left|\left(H_{l}-z\right)^{-1}\right| 0\right\rangle\right),
$$

so

$$
G(z)=\lim _{l \rightarrow \infty} G_{l}(z) \text { for } \operatorname{Im} z>0 .
$$

In Sect 2 we will use the supersymmetric replica trick [15-18] to rewrite $G_{l}(z)$ as a two-point function of a one-dimensional supersymmetric field theory. We will introduce a supersymmetric transfer matrix and do explicitly the integration over the anticommuting variables. This will give us

$$
G_{l}(z)=2 i \int_{0}^{\infty}\left\{\left[(T B(z))^{l} 1\right]\left(r^{2}\right)\right\}^{2} \beta\left(r^{2} ; z\right) r d r,
$$

where $\beta(r ; z)=h(r) e^{i z r}, B(z)$ denotes the operator multiplication by $\beta(\cdot ; z)$, and $T$ is the operator given by

$$
(T f)\left(r^{2}\right)=-2 \int_{0}^{\infty} J_{0}(r s) f^{\prime}(s) s d s
$$

where $J_{0}$ is the Bessel function of order zero. This operator is studied in Sect. 3.

Since the proof of Theorem 1.5 is simpler, we give it first on Sect. 4. Recall $G_{l}(z) \rightarrow$ $G(z)$ as $l \rightarrow \infty$ for $\operatorname{Im} z>0$. It will be easy to see that under the hypothesis of 
Theorem $1.5 G_{l}(z)$ can be analytically continued to $\operatorname{Im} z+\alpha>0$ and (1.1) still holds. We show that (1.1) yields bounds on $G_{l}(z)$, uniformly on $l$, so an application of Vitali's Theorem gives Theorem 1.5.

Section 5 contains the proof of Theorem 1.4. We first show that for large $l(T B(z))^{l} 1$ has $n$ derivatives with good decay properties at infinity. This uses the CalderonLions method of complex interpolation. The theorem is stated in Sect. 5 but proved in Sect. 6. In addition, we show that in this Sobolev-type space $T B(z)$ has 1 as an algebraically simple eigenvalue with a gap in the spectrum. If $\xi(\cdot ; z)$ is the corresponding eigenvector, we will conclude that

$$
G(z)=2 i \int_{0}^{\infty} \xi\left(r^{2} ; z\right)^{2} \beta\left(r^{2} ; z\right) r d r .
$$

Since our estimates will have uniformity properties in $z$, we will be able to let $\eta=\operatorname{Im} z \downarrow 0$ and obtain the conclusions of Theorem 1.4.

Corollary 1.2 is proven in Sect. 7 .

Notes. 1) If $d v / d v$ has an analytic continuation to a strip with decay at infinity, analyticity of the density of states can be derived [31] from formula (IX.5) in [32] and by the methods [29] of [8].

2) Rene Carmona has shown us a manuscript by March and Sznitman [30] with related results. In particular they obtain formula (1.1) by probabilistic methods.

\section{A Supersymmetric Transfer Matrix}

The supersymmetric replica trick [15-18] says that, if $x_{1}, x_{2} \in \Lambda_{l}, \operatorname{Im} z>0$,

$$
\begin{aligned}
G_{l}\left(x_{1}, x_{2} ; z\right) & =\left\langle x_{1}\left|\left(H_{l}-z\right)^{-1}\right| x_{2}\right\rangle \\
& =i \int \psi\left(x_{1}\right) \psi\left(x_{2}\right) \exp \left\{-i \sum_{x=-l}^{l} \Phi(x) \cdot\left[\left(H_{l}-z\right) \Phi\right](x)\right\} D_{l} \Phi,
\end{aligned}
$$

where $\Phi(x)=(\phi(x), \psi(x), \bar{\psi}(x)), \phi(x) \in R^{2}, \psi(x), \bar{\psi}(x)$ are anticommuting "variables" (i.e., elements of a Grassman algebra),

$$
\Phi(x) \cdot \Phi(y)=\phi(x) \cdot \phi(y)+\frac{1}{2}(\tau(x) \psi(y)+\bar{\psi}(y) \psi(x)),
$$

and

$$
D_{l} \Phi=\prod_{x=-l}^{l} d \Phi(x), \quad \text { where } \quad d \Phi(x)=\frac{1}{\pi} d \psi(x) d \psi(x) d^{2} \phi(x)
$$

(see $[29,18,13,20,21,22])$. Notice that $\int e^{-\Phi(x) \cdot \Phi(x)} d \Phi(x)=1$.

Since we are working with a finite lattice the above formula is fully rigorous. To compute functions of $\psi, \tau$ we expand in power series that terminate after a finite number of terms due to the anticommutativity. All $\{\psi(x), \bar{\psi}(x) ; x=-l, \ldots, l\}$ anticommute. The linear functional denoted by integration against $d \psi(x) d \psi(x)$ (it is not an actual integral) is defined by

$$
\int\left(a_{0}+a_{1} \psi(x)+a_{2} \Psi(x)+a_{3} \bar{\psi}(x) \psi(x)\right) d \bar{\psi}(x) d \psi(x)=-a_{3} .
$$

To simplify our notation, we will write $\Phi(x)^{2}=\Phi(x) \cdot \Phi(x), \phi(x)^{2}=\phi(x) \cdot \phi(x)$. 
Recalling the definition of $H_{l}$ we have

$$
\begin{aligned}
G_{l}\left(x_{1}, x_{2} ; z\right)= & i \int \psi\left(x_{1}\right) \psi\left(x_{2}\right) \exp \left\{-i \sum_{x=-l}^{l} V(x) \Phi(x)^{2}+i z \sum_{x=-l}^{l} \Phi(x)^{2}\right. \\
& \left.-i \sum_{x=-l}^{l-1} \Phi(x) \cdot \Phi(x+1)\right\} D_{l} \Phi .
\end{aligned}
$$

Let us first assume that $\int|v| d v(v)<\infty$. This implies that $h$ is continuously differentiable with a bounded derivative. Since in this case

$$
\begin{aligned}
\int e^{i v \Phi^{2}} d v(v) & =\int e^{-i v\left(\phi^{2}+\varangle \psi\right.} d v(v) \\
& =\int e^{-u \phi^{2}}(1-i v \psi \psi) d v(v)=h\left(\phi^{2}\right)+h^{\prime}\left(\phi^{2}\right) \psi \psi=h\left(\phi^{2}+\varangle \psi\right)=h\left(\Phi^{2}\right),
\end{aligned}
$$

we can average over the random potential in (2.1) to obtain

$$
\mathbb{E}\left(G_{l}\left(x_{1}, x_{2} ; z\right)\right)=i \int \psi\left(x_{1}\right) \bar{\psi}\left(x_{2}\right) \prod_{x=-l}^{l} \beta\left(\Phi(x)^{2} ; z\right) \exp \left\{-i \sum_{x=-l}^{l-1} \Phi(x) \cdot \Phi(x+1)\right\} D_{l} \Phi,
$$

where $\beta(r ; z)=h(r) e^{i z r}$.

By an approximation argument we have

Theorem 2.1. Let the characteristic function $h$ be absolutely continuous with a bounded derivative. Then (2.2) holds for $\operatorname{Im} z>0$.

Since in this article we are interested in the density of states we will now take $x_{1}=x_{2}=0$, but our methods work for general $x_{1}, x_{2}$ and give exponential decay for $\lim \mathbb{E}\left(G\left(x_{1}, x_{2} ; E+i \eta\right)\right)$.

$\eta 10$

So let

$$
\begin{aligned}
G_{l}(z)= & \mathbb{E}\left(G_{l}(0,0 ; z)\right)=i \int \psi(0) \bar{\psi}(0) \prod_{x=l}^{l} \beta\left(\Phi(x)^{2} ; z\right) \\
& \cdot \exp \left\{-i \sum_{x=-l}^{l-1} \Phi(x) \cdot \Phi(x+1)\right\} D_{l} \Phi
\end{aligned}
$$

We now introduce a supersymmetric transfer matrix: let

$$
\mathbf{T}\left(\Phi_{1}, \Phi_{2}\right)=e^{-\imath \Phi_{1} \Phi_{2}}
$$

and let us define the operator $\mathbf{T}$ on supersymmetric functions (e.g., [20]) by

$$
(\mathbf{T} F)\left(\Phi_{1}^{2}\right)=\int \mathbf{T}\left(\Phi_{1}, \Phi_{2}\right) F\left(\Phi_{2}^{2}\right) d \Phi_{2}
$$

Let us denote by $B(z)$ the operator multiplication by $\beta(\cdot ; z)$, i.e.,

$$
(B(z) F)\left(\Phi^{2}\right)=\beta\left(\Phi^{2} ; z\right) F\left(\Phi^{2}\right) .
$$

Then (2.3) can be rewritten as

$$
G_{l}(z)=i \int \psi(0) \psi(0) \beta\left(\Phi(0)^{2} ; z\right)\left\{\left[(\mathbf{T} B(z))^{l} 1\right]\left(\Phi(0)^{2}\right)\right\}^{2} d \Phi(0) .
$$

We now perform the integration over the anticommuting variables $\psi(0), \bar{\psi}(0)$ and 
obtain

$$
G_{l}(z)=\frac{i}{\pi} \int \beta\left(\phi(0)^{2} ; z\right)\left\{\left[(T B(z))^{l} 1\right]\left(\phi(0)^{2}\right)\right\} d^{2} \phi(0),
$$

where

$$
(T f)\left(\phi_{1}^{2}\right)=-\frac{1}{\pi} \int e^{-i \phi_{1} \phi_{2}} f^{\prime}\left(\phi_{2}^{2}\right) d^{2} \phi_{2} .
$$

Here we used the fact that if

$$
F\left(\Phi^{2}\right)=f\left(\phi^{2}\right)+f^{\prime}\left(\phi^{2}\right) \psi \psi
$$

then

$$
(\mathbf{T} F)\left(\Phi^{2}\right)=(T f)\left(\phi^{2}\right)+(T f)^{\prime}\left(\phi^{2}\right) \Psi \psi .
$$

If we now change to polar coordinates (2.4) and (2.5) become

$$
G_{l}(z)=2 i \int_{0}^{\infty}\left\{\left[(T B(z))^{l} 1\right]\left(r^{2}\right)\right\}^{2} \beta\left(r^{2} ; z\right) r d r
$$

and

$$
(T f)\left(r^{2}\right)=-2 \int_{0}^{\infty} J_{0}(r s) f^{\prime}\left(s^{2}\right) s d s
$$

where

$$
J_{0}(s)=\frac{1}{2 \pi} \int_{0}^{2 \pi} e^{-i s \cos \theta} d \theta
$$

is the Bessel function of order zero.

\section{Some Harmonic Analysis on $[0, \infty)$}

We will now study the operator $T$ given by (2.7). By an integration by parts,

$$
(T f)\left(r^{2}\right)=f(0)+(R f)\left(r^{2}\right)
$$

where

$$
(R f)\left(r^{2}\right)=r \int_{0}^{\infty} J_{-1}(r s) f\left(s^{2}\right) d s .
$$

We recall that the Bessel functions of integral order $n$ can be defined by

$$
\begin{aligned}
& J_{n}(s)=(-1)^{n} s^{n}\left(\frac{d}{s d s}\right)^{n} J_{0}(s), n=0,1, \ldots, \\
& J_{n}(s)=(-1)^{n} J_{-n}(s) \text { for } n=-1,-2, \ldots,
\end{aligned}
$$

where $J_{0}(s)$ is given by (2.8).

$T$ and $R$ can be expressed in terms of Hankel transforms, which are defined by 
(e.g., $[23,24])$

$$
H_{n}(g)(r)=\int_{0}^{\infty}(r s)^{1 / 2} J_{n}(r s) g(s) d s
$$

for $n \in \mathbb{Z}$.

It is easy to see that $\left\|H_{n}(g)\right\|_{\infty} \leqq 2 / \pi\|g\|_{1}$, and there is a Plancherel theorem for Hankel transforms [23] on $L^{2}([0, \infty), d r):\left\|H_{n}(g)\right\|_{2}=\|g\|_{2}$. It follows from the Riesz convexity theorem that one has a Hausdorff-Young inequality for Hankel transforms:

$$
\left\|H_{n}(g)\right\|_{p^{\prime}} \leqq\|g\|_{p} \quad \text { for } \quad 1 \leqq p \leqq 2, \frac{1}{p}+\frac{1}{p^{\prime}}=1 .
$$

Thus (2.7) and (3.2) can be rewritten as

$$
\begin{aligned}
& r^{1 / 2}(T f)\left(r^{2}\right)=-2 H_{0}\left(s^{1 / 2} f^{\prime}\left(s^{2}\right)\right)(r), \\
& r^{-1 / 2}(R f)\left(r^{2}\right)=H_{-1}\left(s^{-1 / 2} f\left(s^{2}\right)\right)(r) .
\end{aligned}
$$

We have the following general formula for derivatives of Hankel transforms [24]:

$$
r^{n+1 / 2}\left(\frac{d}{r d r}\right)^{m}\left(r^{m-n-1 / 2} g(r)\right)=H_{n}\left((-s)^{m}\left[H_{n-m}(g(t))(s)\right]\right)(r)
$$

for $n=0,1,2, \ldots$, and also for $n=-1$ if $g(0)=0$. Thus

$$
(-2)^{m} r^{m+k-1 / 2}(Q f)^{(m)}\left(r^{2}\right)=(-2)^{k} H_{m+k-1}\left(s^{m+k-1 / 2} f^{(k)}\left(s^{2}\right)\right)(r)
$$

holds with $Q=R$ for $m=0,1,2, \ldots, k=0,1,2, \ldots$, and for $Q=T$ with $m=0,1$, $2, \ldots, k=0,1,2, \ldots$, and $m+k \geqq 1$.

So we are led to define the Hilbert spaces:

$$
\mathscr{H}_{0}=\left\{f:[0, \infty) \rightarrow \mathbb{C} \text { measurable; }\|f\|_{0}=\left\|r^{-1 / 2} f\left(r^{2}\right)\right\|_{2}<\infty\right\},
$$

$\mathscr{H}_{n}=\{f:[0, \infty) \rightarrow \mathbb{C}$ continuous; $f$ is $(n-1)$-times differentiable on $(0, \infty)$ with $f^{(n-1)}$ absolutely continuous with

$$
\left.\|\mid f\|_{n}^{2}=\sum_{m=1}^{n} \sum_{k=0}^{m}\left\|2^{k} r^{m-1 / 2} f^{(k)}\left(r^{2}\right)\right\|_{2}^{2}<\infty\right\}
$$

for $n=1,2, \ldots$, and

$$
\mathscr{H}_{0}^{0}=\mathscr{H}_{0}, \mathscr{H}_{n}^{0}=\left\{f \in \mathscr{H}_{n} ; f(0)=0\right\} \text { for } n=1,2, \ldots
$$

It follows from (3.5) that $T$ is a unitary operator on $\mathscr{H}_{n}$ for $n=1,2, \ldots$, and $R$ is unitary on $\mathscr{H}_{n}^{0}$ for $n=0,1,2, \ldots$ In addition (3.1) says that $T=R$ on $\mathscr{H}_{n}^{0}$ for $n \geqq 1$; in particular $T$ leaves $\mathscr{H}_{n}^{0}$ invariant.

Let us now denote by $B$ the operator multiplication by $\beta \in \mathscr{H}_{1}$. Then $(T B)^{l} 1$ is well defined. It also follows that $r^{-1 / 2} \beta\left(r^{2}\right) \in L^{1}$, so by (3.4) $R \beta$ is well defined and a bounded continuous functions with $(R \beta)(0)=0$. Thus if we apply (3.1) $l$ times we get

$$
(T B)^{l} 1=\left(I+R B+(R B)^{2}+\cdots+(R B)^{l}\right) 1 .
$$


For later use we rewrite (3.6) as

$$
(T B)^{l} 1=1+R B+\left(I+R B+\cdots+(R B)^{l-2}\right)(R B)^{2} 1,
$$

and

$$
(T B)^{l} 1=(T B) 1+\left(I+R B+\cdots+(R B)^{l-2}\right)(R B)^{2} 1 .
$$

If only assume that $\beta\left(r^{2}\right) \in L^{\infty}$, we still have $R B$ as a bounded operator on $\mathscr{H}_{0}$. The following lemmas will be of importance.

Lemma 3.1. Let $\beta\left(r^{2}\right) \in L^{p}([0, \infty), d r)$, where $2<p \leqq \infty$. Then $\left\|(R B)^{2}\right\|_{\mathscr{H}_{0}} \leqq$ $\left\|\beta\left(r^{2}\right)\right\|_{p}^{2}$.

Proof. Let $f \in \mathscr{H}_{0}$. Then

$$
\begin{aligned}
\left\|(R B)^{2} f\right\|_{0} & =\|B R B f\|_{0}=\left\|r^{-1 / 2} \beta\left(r^{2}\right)(R B f)\left(r^{2}\right)\right\|_{2} \\
& \leqq\left\|\beta\left(r^{2}\right)\right\|_{p}\left\|r^{-1 / 2}(R B f)\left(r^{2}\right)\right\|_{(1 / 2-1 / p)^{-1}} \\
& \leqq\left\|\beta\left(r^{2}\right)\right\|_{p}\left\|r^{-1 / 2}(B f)\left(r^{2}\right)\right\|_{(1 / 2+1 / p)^{-1}} \\
& =\left\|\beta\left(r^{2}\right)\right\|_{p}\left\|r^{-1 / 2} \beta\left(r^{2}\right) f\left(r^{2}\right)\right\|_{(1 / 2+1 / p)^{-1}} \\
& \leqq\left\|\beta\left(r^{2}\right)\right\|_{p}^{2}\left\|r^{-1 / 2} f\left(r^{2}\right)\right\|_{2}=\left\|\beta\left(r^{2}\right)\right\|_{p}^{2}\|f\|_{0} .
\end{aligned}
$$

Lemma 3.2. Suppose $\beta$ is a continuous function such that $\left(1+r^{2}\right)^{\gamma / 2} \beta\left(r^{2}\right)$ is bounded for some $\gamma>0$. Then $(R B)^{2} 1 \in \mathscr{H}_{0}$.

Proof. It follows that $r^{1 / 2} \beta\left(r^{2}\right) \in L^{q}$ for all $1<q_{1}<q<2$, where $q_{1}$ depends only on $\gamma$, and $\beta\left(r^{2}\right) \in L^{p}$ for all large $p$. Thus $r^{-1 / 2}(R \beta)\left(r^{2}\right) \in L^{q^{\prime}}$, where $1 / q+1 / q^{\prime}=1$, and $r^{-1 / 2} \beta\left(r^{2}\right)(R \beta)\left(r^{2}\right) \in L^{2}$.

\section{Proof of Theorem $\mathbf{1 . 5}$}

We first assume that $h$ is also absolutely continuous with $h^{\prime}$ bounded, so Theorem 2.1 applies and we have, from (2.6) and (3.6), that

$$
G_{l}(z)=2 i \int_{0}^{\infty}\left\{\left[\sum_{k=0}^{l}\left[(R B(z))^{k} 1\right]\left(r^{2}\right)\right\}^{2} \beta\left(r^{2} ; z\right) r d r \text { for } \operatorname{Im} z>0 .\right.
$$

By an approximation argument we can now extend (4.1) to $h$ as in the hypothesis of Theorem 1.5.

Since $\beta\left(r^{2} ; z\right)=h\left(r^{2}\right) e^{i z r^{2}}$, and $e^{\alpha r^{2}} h\left(r^{2}\right)$ is bounded with $\alpha>0$, we can use the righthand side of (4.1) to analytically continue $G_{l}(z)$ to $\operatorname{Im} z+\alpha>0$.

Since $|h(t)|<1$ for all $t \neq 0$, there exists $2<p<\infty$ such that $\int_{0}^{\infty}\left(\left.h\left(r^{2}\right)\right|^{p} d r<1\right.$. Since $e^{\tau r^{2}} h\left(r^{2}\right) \in L^{p}$ for $\tau<\alpha$, we can select $0<\tau<\alpha$ such that $\left\|e^{\tau r^{2}} h\left(r^{2}\right)\right\|_{p}<1$.

It now follows from (4.1), (3.7), Lemmas 3.1 and 3.2 that $G_{l}(z)$ is uniformly bounded in $l$ and in $z$ for $\operatorname{Im} z+\tau>0$. It follows from Vitali's Theorem that $G(z)$ is analytic for $\operatorname{Im} z+\tau>0$. 


\section{Proof of Theorem 1.4}

Under the hypothesis of Theorem $1.4, \beta(r ; z)=h(r) e^{i z r}$ is $(n-1)$-times differentiable for $r \geqq 0$ with $\beta^{(n-1)}(r ; z)$ absolutely continuous, and, if $\operatorname{Im} z \geqq 0,\left(1+r^{2}\right)^{\gamma / 2} \beta^{(j)}\left(r^{2} ; z\right)$ is bounded, $j=0,1, \ldots, n$, for some $\gamma>0$. As before $B(z)$ will denote the operator multiplication by $\beta(\cdot ; z)$. Notice that $B(z)$ is a bounded operator on $\mathscr{H}_{m}$, leaving $\mathscr{H}_{m}^{0}$ invariant, for $\operatorname{Im} z \geqq 0$ and $m=0,1, \ldots, n$.

We will need more. We will need that applying $R B(z)$ repeatedly takes $\mathscr{H}_{0}$ to $\mathscr{H}_{n}^{0}$.

Theorem 5.1. Let $\beta(r)$ be $(n-1)$-times differentiable with $\beta^{(n-1)}(r)$ absolutely continuous, such that $\left(1+r^{2}\right)^{\gamma / 2} \beta^{(j)}\left(r^{2}\right)$ is bounded, $j=0,1, \ldots, n$, for some $\alpha>0$. Let $B$ be the operator multiplication by $\beta$. Then there exists $k_{0}$ depending only on $\gamma$, such that for all $k \geqq k_{0},(R B)^{k}$ is a bounded operator from $\mathscr{H}_{0}$ to $\mathscr{H}_{n}^{0}$. Furthermore, if $\beta(r ; z)=$ $\beta(r) e^{i z r}$ and $B(z)$ is the corresponding multiplication operator, the norm of $(R B(z))^{k}$ as an operator from $\mathscr{H}_{0}$ to $\mathscr{H}_{n}^{0}$ is uniformly bounded for $\operatorname{Im} z \geqq 0$ and bounded $\operatorname{Re} z$.

If $\gamma>1$ (e.g., if the probability distribution $v$ is the uniform distribution on a bounded interval) it is not hard to prove this theorem. But for small $\gamma$ it requires the Calderon-Lions method of complex interpolation, so we will postpone it to the next section.

Let $g(t)$ be a real valued $C^{\infty}$ function with compact support on $\mathbb{R}$ such that $g(t)=1$ for $|t| \leqq 1$. Let $h_{1}(t)=g(t) h(t), h_{2}(t)=h(t)-h_{1}(t)$, and let $\beta_{j}(r ; z)=h_{j}(r) e^{i z r}$, $j=1,2$. Then

$$
\beta(r ; z)=\beta_{1}(r ; z)+\beta_{2}(r ; z) \quad \text { and } \quad \beta_{1}(r ; z) \in \mathscr{H}_{n}, \beta_{2}(r ; z) \in \mathscr{H}_{0}
$$

for $\operatorname{Im} z \geqq 0$.

Recall that (3.8) holds for $\operatorname{Im} z>0$, so we have

$$
(T B(z))^{l} 1=T \beta_{1}(z)+R \beta_{2}(z)+\left(I+R B(z)+\cdots+(R B(z))^{l-2}(R B(z))^{2} 1\right.
$$

for $\operatorname{Im} z>0$.

By Lemma 3.2, $(R B(z))^{2} 1 \in \mathscr{H}_{0}$ for $\operatorname{Im} z \geqq 0$, and the right-hand side of (5.1) is well defined for $\operatorname{Im} z \geqq 0$.

Now let us pick $k_{0}$ from Theorem 5.1. It follows that

$$
\begin{aligned}
(T B(z))^{l+k_{0}} 1= & (T B)^{k_{0}} T \beta_{1}(z)+(R B(z))^{k_{0}}\left[R \beta_{2}(z)\right. \\
& +\left(T+R B(z)+\cdots+(R B(z))^{l-2}\right)\left(R B(z)^{2} 1\right]
\end{aligned}
$$

is in $\mathscr{H}_{n}$ for $\operatorname{Im} z>0$ and the right-hand side is a continuous function of $z, \operatorname{Im} z \geqq 0$, with values in $\mathscr{H}_{n}$. We have proved the first part of

Lemma 5.2. There exists $l_{0}$ such that for $l \geqq l_{0}(T B(z))^{l} 1 \in \mathscr{H}_{n}$ for $\operatorname{Im} z>0$, is a continuous function of $z$ with values in $\mathscr{H}_{n}$, and can be extended by continuity to $\operatorname{Im} z \geqq 0$. Furthermore $\xi(z)=\lim _{l \rightarrow \infty}(T B(z))^{l} 1$ exists in $\mathscr{H}_{n}$ for $\operatorname{Im} z \geqq 0$, the convergnece being uniform in $\operatorname{Im} z \geqq 0$ with bounded $\operatorname{Re} z$.

Proof. The lemma follows from (5.2) and Lemma 3.1. Just notice that $\left\|\beta\left(r^{2} ; z\right)\right\|_{p} \leqq\left\|h\left(r^{2}\right)\right\|_{p}$ for $\operatorname{Im} z \geqq 0$, and that $\left\|h\left(r^{2}\right)\right\|_{p}<1$ for $p$ large enough. 
Notice that Lemma 3.2 and (2.6) tell us that

$$
G(z)=2 i \int_{0}^{\infty} \xi\left(r^{2} ; z\right)^{2} \beta\left(r^{2} ; z\right) r d r
$$

and $G(z)$ is a continuous function of $z$ for $\operatorname{Im} z \geqq 0$. This is Theorem 1.4 for $n=1$.

Lemma 3.2 and its proof also tell us that $T B(z) \xi(z)=\xi(z)$ and $\xi(0 ; z)=1$. In fact we have more:

Lemma 5.3. Let $\operatorname{Im} z \geqq 0$. Then 1 is an algebraically simple eigenvalue for $T B(z)$ in $\mathscr{H}_{n}$ with corresponding unique eigenvector $\xi(z)$ normalized by $\xi(0 ; z)=1$. Furthermore, the direct sum $\mathscr{H}_{n}=\mathbb{C} \xi(z) \oplus \mathscr{H}_{n}^{0}$ diagonalizes $T B(z)$ in the form $T B(z)=\delta_{0} \xi(z) \oplus R B(z)$, where $\delta_{0}(f)=f(0)$. In addition, the operator norm of $(R B(z))^{2}$ in $\mathscr{H}_{n}^{0}$ is bounded by a constant $<1$ uniformly in $\operatorname{Im} z \geqq 0$ and bounded $\operatorname{Re} z$.

Proof. If $f \in \mathscr{H}_{n}$, then $\left.f=f(0) \xi(z)+[f-f(0) \xi(z))\right]$ and $f-f(0) \xi(z) \in \mathscr{H}_{n}^{0}$. Thus $\mathscr{H}_{n}=\mathbb{C} \xi(z) \oplus \mathscr{H}_{n}^{0}$. The lemma now follows from Lemmas 5.2, 3.1, and Theorem 5.1.

To finish the proof of Theorem 1.4 for $n \geqq 2$, we must show that $G(E+i 0)$ is a $C^{n-1}$ function of $E$. From (5.3) we have

$$
G(E+i 0)=2 i\langle M \xi(E), B(E) M \xi(E)\rangle,
$$

where

$$
\langle u, v\rangle=\int_{0}^{\infty} u\left(r^{2}\right) v\left(r^{2}\right) r^{-1} d r
$$

is a continuous bilinear form on 0 and $M$ is the operator multiplication by the function $r^{1 / 2}$, i.e., $(M u)\left(r^{2}\right)=r u\left(r^{2}\right)$.

Let us fix $E_{0} \in R, \delta>0$, and let

$$
\tau_{0}^{2}=\sup \left\{\left\|(R B(E))^{2}\right\|_{\mathscr{H}_{n}^{0}} ;\left|E-E_{0}\right|<\delta\right\}<1
$$

by Lemma 5.3. Let $Y$ denote the circle $\left\{z \in \mathbb{C} ;|z-1|=\frac{1}{2}\left(1-\tau_{0}\right)\right\}, \xi_{0}=\xi\left(E_{0}\right)$. Then

$$
\xi(E)=\frac{1}{2 \pi i} \int_{\Upsilon}(z-T B(E))^{-1} d z \xi_{0}
$$

for $\left|E-E_{0}\right|<\delta$.

Since $E \rightarrow T B(E)$ is a continuous function with values in $\mathscr{L}\left(\mathscr{H}_{n}\right)$, the space of bounded linear operators on $\mathscr{H}_{n}$, it follows from (5.5) that $E \rightarrow \xi(E)$ is continuous with values in $\mathscr{H}_{n}$.

Now, $T B(E)$ is not differentiable as a function with values in $\mathscr{L}\left(\mathscr{H}_{n}\right)$, but it is as a function with values in $\mathscr{L}\left(\mathscr{H}_{n}, \mathscr{H}_{n-2}\right)$, the space of bounded linear operators from $\mathscr{H}_{n}$ to $\mathscr{H}_{n-2}$, as long as $n \geqq 2$, and $d / d t T B(E)=i T M^{2} B(E)$. So it follows from (5.5) that $\xi(E)$ is continuously differentiable with $d \xi / d E(E) \in \mathscr{H}_{n-2}$.

More generally, if $2 k \leqq n, T B(E)$ is $k$ times continuously differentiable with

$$
\frac{d^{k}}{(d E)^{k}} T B(E)=i^{k} T M^{2 k} B(E) \in \mathscr{L}\left(\mathscr{H}_{n}, \mathscr{H}_{n-2 k}\right) \text {, }
$$


and it follows from (5.5) that $\xi(E)$ is $k$-times differentiable with $d^{k} / d E^{k} \xi(E) \in \mathscr{H}_{n-2 k}$.

It now follows from (5.4) that $G(E+i 0)$ is a $C^{2 k}$ function of $E$ if $2 k \leqq n$.

But we can do better, in fact we will show $G(E+i 0)$ is $C^{n-1}$.

To do so notice that $R$ is self-transpose with respect to $\langle$,$\rangle on \mathscr{H}_{0}$, i.e.,

$$
\langle R f, g\rangle=\langle f, R g\rangle \text { for } f, g \in \mathscr{H}_{0} .
$$

Similarly, $B(E)^{t}=B(E)$, the transpose being with respect to $\langle$,$\rangle on \mathscr{H}_{0}$, so

$$
(R B(E))^{t}=B(E) R,\left[(z-R B(E))^{-1}\right]^{t}=(z-B(E) R)^{-1} .
$$

We also recall that $T=R$ if $f(0)=0$.

From (5.4) and (5.5) we get, for $\left|E-E_{0}\right|<\delta$,

$$
G(E+i 0)=\frac{1}{2 \pi^{2} i} \int_{\Upsilon} d z \int_{\Upsilon} d z^{\prime}\left\langle M K(z, E) \xi_{0}, B(E) M K\left(z^{\prime}, E\right) \xi_{0}\right\rangle,
$$

where $K(z, E)=(z-T B(E))^{-1}$.

If $n=2$, it is not hard to see that since $\xi_{0} \in \mathscr{H}_{2}$,

$$
\begin{aligned}
\frac{d}{d E} G(E+i 0)= & \frac{1}{2 \pi^{2}} \int_{Y} d z \int_{Y} d z^{\prime}\left\{2\left\langle K(z, E) T i M^{2} B(E) K(z, E) \xi_{0}, M^{2} B(E) K\left(z^{\prime}, E\right) \xi_{0}\right\rangle\right. \\
& \left.+\left\langle M^{2} K(z, E) \xi_{0}, i M^{2} B(E) K\left(z^{\prime}, E\right) \xi_{0}\right\rangle\right\}
\end{aligned}
$$

a continuous function of $E$.

The same procedure can be used for general $n$. For an operator valued function $A(E)$, let $\Delta_{e} A(E)=1 / e(A(E+e)-A(E))$.

When we compute $\lim _{e \rightarrow 0} \Delta_{e}\left(d^{k} / d E^{k}\right) G(E+i 0)$, we must move some operators from one side to the other of the bilinear form $\langle$,$\rangle using the transposed operators. We$ illustrate the procedure in the following term that appears in $\Delta_{e}(d / d E) G(E+i 0)$ : $2\left\langle M K(z, E+e) T\left(\Delta_{e} B(E)\right) K(z, E) T i M^{2} B(E+e) K(z, E+e) \xi_{0}, B_{E+e} M K\left(z^{\prime}, E+e\right) \xi_{0}\right\rangle$.

In this case $\xi_{0} \in \mathscr{H}_{3}$. We cannot just take the limit as $e \rightarrow 0$ for the vector appearing on the right-hand side of $\langle$,$\rangle because the vector to which the last$ operator $T$ would be applied would not necessarily be in $\mathscr{H}_{0}$ since $\Delta_{e} B(E) \rightarrow$ $i M^{2} B(E)$, and we may only have $\xi_{0} \in \mathscr{H}_{3}$. But (5.6) can be rewritten as

$$
\begin{gathered}
2\left\langle\left(\Delta_{e} B(E)\right) K(z, E) T i M^{2} B(E+e) K(z, E+e) \xi_{0},\right. \\
\left.T K(z, E+e)^{t} M^{2} B(E+e) K\left(z^{\prime}, E+e\right) \xi_{0}\right\rangle .
\end{gathered}
$$

The rearrangement is legitimate since all vectors are in the right spaces. We can now take the limit as $e \rightarrow 0$ and obtain

$$
2\left\langle i M B(E) K(\xi, E) T i M^{2} B(E) K(z, E) \xi_{0}, M T K(E, z)^{t} M^{2} B(E) K\left(z^{\prime}, E\right) \xi_{0}\right\rangle .
$$

The same procedure can be applied to all terms appearing in $\Delta_{e}$ $\left(d^{k} / d E^{k}\right) G(E+i 0), \quad k \leqq n-2, \quad$ to give existence and continuity of $\left(d^{k+1} / d E^{k+1}\right) G(E+i 0)$. This proves Theorem 1.4. 


\section{Proof of Theorem 5.1}

The proof will proceed by induction on $n$.

If $n=0$, there is nothing to prove since $R B$ is a bounded operator from $\mathscr{H}_{0}$ to $\mathscr{H}_{0}$ (notice that the theorem makes sense for $n=0$, the hypothesis being simply that $\beta(r)$ is a bounded measurable function).

So let us assume the theorem is true for $n-1, n \geqq 1$; we will prove the theorem is then true for $n$.

We are going to use repeatedly the Calderon-Lions interpolation theorem $[25,26]$. We will use the notation $V_{t}, 0 \leqq t \leqq 1$, for the interpolating spaces between $V_{0}$ and $V_{1}$. We will write $V_{t}^{(1)}=V_{t}, V_{t}^{(m)}=$ the $t^{t h}$ interpolating space between $V_{t}^{(m-1)}$ and $V_{1}$. In what follows $S: V \rightarrow W$ or $V \stackrel{S}{\rightarrow} W$ mean that $S$ is a bounded operator from $V$ to $W$. For all spaces $V_{0}$ and $V_{1}$ between which we will interpolate we will have I: $V_{1} \rightarrow V_{0}$. We start by introducing the following spaces:

$$
X_{0}=Y_{0}=Z_{0}=\mathscr{H}_{0}, \quad Z_{1}=\mathscr{H}_{n}^{0},
$$

and

$$
X_{1}=\left\{f:[0, \infty) \rightarrow \mathbb{C} \text { measurable; }\left\|\left(1+r^{2}\right)^{n / 2} r^{-1 / 2} f\left(r^{2}\right)\right\|_{2}<\infty\right\},
$$

$Y_{1}=\left\{f:[0, \infty) \rightarrow \mathbb{C}(n-1)\right.$-times differentiable on $(0, \infty)$ with $f^{(n-1)}$ absolutely continuous; $\left.\sum_{k=0}^{n}\left\|r^{k-1 / 2} f^{(k)}\left(r^{2}\right)\right\|_{2}^{2}<\infty\right\}$.

We can identify the interpolating spaces $X_{t}[26]$ :

$$
X_{t}=\left\{f:[0, \infty) \rightarrow \mathbb{C} \text { measurable; }\left\|\left(1+r^{2}\right)^{n t / 2} r^{-1 / 2} f\left(r^{2}\right)\right\|_{2}<\infty\right\} .
$$

From (3.5) we have

$$
X_{0} \stackrel{R}{\rightarrow} Y_{0} \stackrel{R}{\rightarrow} X_{0}, \quad X_{1} \stackrel{R}{\rightarrow} Y_{1} \stackrel{R}{\rightarrow} X_{1},
$$

so we conclude that $X_{t} \stackrel{R}{\rightarrow} Y_{t} \stackrel{R}{\rightarrow} X_{t}$ for all $t \in[0,1]$. Recall $R^{2}=I$.

Let us write $\sigma=\gamma / n$ and notice that $X_{0} \stackrel{R}{\rightarrow} X_{0} \stackrel{B}{\rightarrow} X_{\sigma} \stackrel{R}{\rightarrow} Y_{\sigma}$.

We now interpolate between the $Y$ 's and the $Z$ 's. Let $S(\zeta)=e^{\zeta^{2}} B\left(1+r^{2}\right)^{(\sigma-\zeta) n / 2}$ for $\operatorname{Re} \zeta \in[0,1]$. Then $S(0): Y_{0} \rightarrow Z_{0}$ and $S(1)=Y_{1} \rightarrow Z_{1}$ by the hypothesis on $\beta$. It is easy to see that $S(\zeta)$ satisfies the hypothesis of Theorem IX. 20 in [25], so we can conclude that $S(t): Y_{t} \rightarrow Z_{t}$ for $t \in[0,1]$. Taking $t=\sigma$, we get $B: Y_{\sigma} \rightarrow Z_{\sigma}$.

We have so far shown that $(R B)^{2}: X_{0} \rightarrow Z_{\sigma}$. Since $(R B)^{2}: Z_{1} \rightarrow Z_{1}$, we have that $(R B)^{4}: X_{0} \rightarrow Z_{\sigma}^{(2)}$. Reiterating the argument, we get that $(R B)^{2 m}: X_{0} \rightarrow Z_{\sigma}^{(m)}$.

Now let $W_{0}=\mathscr{H}_{n-1}^{0}, W_{1}=\mathscr{H}_{n}^{0}$. By the induction hypothesis there exists $k_{1}$ such that $(R B): Z_{0} \rightarrow W_{0}$ and, of course, $(R B)^{k_{1}}: Z_{n} \rightarrow W_{n}$. It follows $(R B)^{k_{1}+2 m}: X_{0} \rightarrow W_{\sigma}^{(m)}$.

Now let $D$ be the operator defined by $(D f)\left(r^{2}\right)=f^{\prime}\left(r^{2}\right)$, and let

$$
V_{t}=\left\{f:[0, \infty) \rightarrow \mathbb{C} \text { measurable; }\left\|r^{n-1+t-1 / 2} f\left(r^{2}\right)\right\|_{2}<\infty\right\},
$$


where $0 \leqq t \leqq 1$. If $k=0,1, \ldots, n-1, D^{k}: W_{0} \rightarrow V_{0}, D^{k}: W_{1} \rightarrow V_{1}$, so it follows that $D^{k}$ : $W_{\sigma}^{(m)} \rightarrow V_{\sigma}^{(m)}$.

But we can identify $V_{\sigma}^{(m)} \quad[26]: V_{\sigma}^{(m)}=\{f: \quad[0, \infty) \rightarrow \mathbb{C}$ measurable; $\left.\left\|r^{n-(1-\sigma)^{m}-1 / 2} f\left(r^{2}\right)\right\|_{2}<\infty\right\}$.

So we choose $m$ such that $(1-\sigma)^{m}<\gamma$. If $f \in W_{\sigma}^{(m)}, f^{(k)} \in V_{\sigma}^{(m)}$ for $k=0,1, \ldots, n-1$. Thus $(B f)^{(k)} \in V_{1}$ for $k=0,1, \ldots, n-1$. It follows from (3.5) that $(R B f)^{(k)} \in V_{1}$ for $k=1, \ldots, n$.

Now let $f \in X_{0}$. Then $(R B)^{k_{1}+2 m} f \in W_{\sigma}^{(m)}$, so

$$
\left((R B)^{k_{1}+2 m+1} f\right)^{(k)} \in V^{1} \text { for } \quad k=1, \ldots, n .
$$

On the other hand, $R B: X_{0} \rightarrow X_{0}$, so

$$
B(R B)^{k_{1}+2 m+1} f \in V_{1} \text {. }
$$

From (6.1) and (6.2) we conclude that $B(R B)^{k_{1}+2 m+1} f \in \mathscr{H}_{n}$, and hence is in $\mathscr{H}_{n}^{0}$, so $(R B)^{k_{1}+2 m+2} f \in \mathscr{H}_{n}^{0}$ for all $f \in X_{0}=\mathscr{H}_{0}$.

If $\beta(r ; z)=\beta(r) e^{i z r}, B(z)$ the corresponding multiplication operator, it is easy to check in the proof that we get the desired uniformity in $z$ for the norm of $(R B(z))^{k}$.

\section{Proof of Corollary 1.2}

Corollary 1.2 follows from

Lemma 7.1. Let $\left(1+|t|^{\alpha}\right) h(t)$ be bounded for some $\alpha>0$ and let $\int|v|^{n+\varepsilon} d v(v)<\infty$ for some $\varepsilon>0$. Then $h$ is $n$-times differentiable and there exists $\delta>0$ such that $(1+|t|)^{\delta} h^{(j)}(t)$ is bounded for $j=0,1, \ldots, n$.

Proof. We will show that there exist $\delta>0$ such that $(1+|t|)^{\delta} h^{(n)}(t)$ is bounded. Let $\chi(v)$ be a $C^{\infty}$ function such that $\chi(v)=v^{n}$ for $|v| \leqq 1, \chi(v)=0$ for $|v| \geqq 2$, and $|\chi(v)| \leqq 2$ for all $v$. For $R>0$ let $\chi_{R}(v)=R^{n} \chi\left(R^{-1} v\right)$.

For any $k \geqq 0$ there exists $C_{k}<\infty$ such that if $\hat{\chi}(t)=\int e^{-i t v} \chi(v) d v,|\hat{\chi}(t)| \leqq$ $C_{k}\left(1+|t|^{k}\right)^{-1}$. It follows that

$$
\left|\hat{\chi}_{R}(t)\right| \leqq C_{k} R^{n+1}\left(1+R^{k}|t|^{k}\right)^{-1} .
$$

Since $h^{(n)}(t)=(-i)^{n} \int v^{n} e^{-i t v} d v(v)$, we have that for $R \geqq 2$

$$
\begin{aligned}
\left|h^{(n)}(t)-(-i)^{n} \int \chi_{R}(v) e^{-i t v} d v(v)\right| & =\left|\int_{|v| \geqq R}\left(v^{n}-\chi_{R}(v)\right) e^{-i t v} d v(v)\right| \\
& \leqq 2 \int_{|v| \geqq R}|v|^{n} d v(v) \leqq 2 R^{-\varepsilon} \int|v|^{n+\varepsilon} d v(v) .
\end{aligned}
$$

We have

$$
\begin{aligned}
\int \chi_{R}(v) e^{-i t v} d v(v)= & (2 \pi)^{-1}\left(\hat{\chi}_{R} * h\right)(t)=(2 \pi)^{-1} \int_{|s| \leqq t / 2} \hat{\chi}_{R}(s) h(t-s) d s \\
& +(2 \pi)^{-1} \int_{|s|>t / 2} \hat{\chi}_{R}(s) h(t-s) d s .
\end{aligned}
$$


We now use (7.1) to estimate each term; we have

$$
\left|\int_{|s| \leqq t / 2} \hat{\chi}_{R}(s) h(t-s) d s\right| \leqq D_{k} R^{n}(1+|t|)^{-\alpha}
$$

and

$$
\left|\int_{|s|>t / 2} \hat{\chi}_{R}(s) h(t-s) d s\right| \leqq C_{k} R^{n+1} \int_{|s|>t / 2}\left(1+R^{k}|s|^{k}\right)^{-1} d s \leqq D_{k}^{\prime} R^{n}(R|t|)^{1-k},
$$

where $D_{k}$ and $D_{k}^{\prime}$ are finite if we take $k>1$.

From (7.2), (7.3), (7.4) and (7.5), we get

$$
\left|h^{(n)}(t)\right| \leqq K_{1} R^{-\varepsilon}+K_{2} R^{n}\left((1+|t|)^{-\alpha}+(R|t|)^{1-k}\right)
$$

with $K_{1}$ and $K_{2}$ finite constants depending on $k>1$. Fix $k$. Then for fixed $t$ pick $R=$ $R(t)=|t|^{-\sigma}$. It is clear from (7.6) that we can pick an appropriate $\sigma>0$ to get the desired result.

Acknowledgements. A. K: wants to express his debt to J. Fernando Perez for their collaboration on which the ideas on supersymmetry and the supersymmetric replica trick that are used in this paper were developed, and for the idea of using a supersymmetric transfer matrix in the one-dimensional case. A. K. also wants to thank Rene Carmona for many enlightening conversations about random potentials. We want to thank Tom Wolff for references $[27,28]$.

M. C. wants to express his gratitude to the University of California, Irvine for the hospitality that made this collaboration possible.

\section{References}

1. Benderskii, M., Pastur, L.: On the spectrum of the one dimensional Schrödinger equation with a random potential. Mat. Sb. 82, 245-256 (1970)

2. Avron, J., Simon, B.: Almost periodic Schrödinger operators, II. The integrated density of states. Duke Math. J. 50, 369-391 (1983)

3. Kirsch, W., Martinelli, F.: On the density of states of Schrödinger operators with a random potential. J. Phys. A15, 2139-2156 (1982)

4. Carmona, R.: Random Schrödinger operators, proceedings of the ecole d'Ete de probabilite XIV, Saint-Flour, 1984 (to appear)

5. Pastur, L.: Spectral properties of disordered systems in one-body approximation, Commun. Math. Phys. 75, 179 (1980)

6. Craig, W., Simon, B.: Subharmonicity of the Lyaponov index. Duke Math. J. 50, 551-560 (1983)

7. LePage, E.: Empirical distribution of the eigenvalues of a Jacobi matrix. In: Probability measures on groups, VII. Lecture Notes Vol. 1064, pp. 309-367 Berlin, Heidelberg, New York: Springer 1983

8. Simon, B., Taylor, M.: Harmonic analysis on $\operatorname{SL}(2, R)$ and smoothness of the density of states in the one dimensional Anderson model. Commun. Math. Phys. 101, 1-19 (1985)

9. Wegner, F.: Bounds on the density of states in disordered systems. Z. Phys. B44, 9-15 (1981)

10. Maier, R.: The density of states of random Schrödinger operators. To appear in the Proceedings of AMS Bowdoin Summer Conference (1984)

11. Lacroix, J.: Products of random matrices with applications to Schrödinger operators. (In preparation)

12. Constantinescu, F., Fröhlich, J., Spencer, T.: Analyticity of the density of states and replica method for random Schrödinger operators on a lattice, J. Stat. Phys. 34, 571-596 (1984)

13. Klein, A., Perez, J. F.: (in preparation)

14. Simon, B.: Kotani theory for one dimensional stochastic Jacobi matrices. Commun. Math. Phys. 89, 227-234 (1983)

15. Parisi, G., Sourlas, N.: Self-avoiding walk and supersymmetry, J. Phys. Lett. 41, L403-L406 (1980) 
16. McKane, A. J.: Reformulation of $n \rightarrow 0$ models using anticommuting scalar fields. Phys. Lett. 76A, 22-24 (1980)

17. Luttinger, J. M.: The asymptotic evaluation of a class of path integrals II. J. Math. Phys. 24, 2070$2073(1983)$

18. Effetov, K. B.: Supersymmetry and the theory of disordered metals. Adv. Phys. 32, 53-127 (1983)

19. Berezin, F. A.: The method of second quantization. New York: Academic Press 1966

20. Klein, A., Landau, L. J., Perez, J. F.: Supersymmetry and the Parisi-Sourias dimensional reduction: A rigorous proof. Commun. Math. Phys. 93, 459-482 (1984)

21. Klein, A.: Supersymmetry and a two-dimensional reduction in random phenomena. Proceedings of the Second Workshop on Quantum Probability and Applications. In: Lecture Notes in Mathematics Berlin, Heidelberg, New York: Springer Vol. 1136, pp. 306-317 (1985)

22. Klein, A., Perez, J. F.: On the density of states for random potentials in the presence of a uniform magnetic field. Nucl. Phys. B251, [FS13] 199-211 (1985)

23. Titchmarsh, E. C.: Introduction to the theory of Fourier integrals. Oxford: Oxford University Press 1948

24. Erderlyi, A.: Tables of Integral Transforms, vol. II. New York: McGraw Hill 1954

25. Reed, M., Simon, B.: Methods of modern mathematical physics, II. Fourier analysis, self-adjointness. New York: Academic Press 1975

26. Stein, E.: Singular integrals and differentiability properties of functions. Princeton: Princeton University Press 1970

27. Zygmund, A.: Trigonometric Series, vol. II, Cambridge: Cambridge University Press 1959

28. Brown, G., Hewitt, E.: Adv. Math. 37, 27-60 (1980)

29. Simon, B.: private communication

30. March, P., Sznitman, A.: Some connections between excursion theory and the discrete random Schrödinger equation, with applications to analyticity and smoothness properties of the density of states in one dimension. (Preprint)

31. Souillard, B.: private communication

32. Kunz, H., Souillard, B.: Sur le spectre des operateurs aux differences finies aleatoires. Commun. Math. Phys. 78, 201-246 (1980)

Communicated by T. Spencer

Received June 24, 1985; in revised form September 23, 1985 
